\title{
Hematoma de la vaina de los rectos en un recién nacido macrosómico después de un parto distócico. A propósito de un caso
}

\author{
Rectus sheath hematoma in a macrosomic neonate following difficult delivery. \\ Case report
}

Dra. Suzan Gunduz $z^{a}$ Dra. Semra Kara ${ }^{b}$, Dra. Ayşe Aktasc ${ }^{c}$ Dr. Abdulkadir Eren ${ }^{b}$ y Dr. Vedat Bakan ${ }^{d}$

\section{RESUMEN}

La macrosomía es un factor de riesgo de traumatismos obstétricos y se asocia con morbimortalidad neonatal. El cefalohematoma y la fractura clavicular son los más frecuentes. Los traumatismos intrabdominales son poco frecuentes. El hematoma de la vaina de los rectos es una acumulación de sangre en la vaina del músculo recto mayor del abdomen. Se asocia con traumatismos, cirugías y tratamiento anticoagulante, en especial en los adultos y las personas de edad avanzada. En este artículo presentamos el caso de un recién nacido de sexo masculino con parto vaginal distócico. Durante el examen físico se observó un hematoma periumbilical de $1 \times 1 \mathrm{~cm}$ y un cefalohematoma parietal de $1 \times 1 \mathrm{~cm}$. En la ecografía abdominal y la tomografía computada del abdomen se observó un hematoma de la vaina del recto derecho de $7 \times 4 \times 2 \mathrm{~cm}$.

Palabras clave: hematoma de la vaina de los rectos, macrosomía fetal, traumatismos obstétricos, recién nacidos.

http:/ / dx.doi.org/10.5546/ aap.2018.e82

Texto completo en inglés:

http:/ / dx.doi.org/10.5546/ aap.2018.eng.e82

Cómo citar: Gunduz S, Kara S, Aktas A, et al.Hematoma de la vaina de los rectos en un recién nacido macrosómico después de un parto distócico. A propósito de un caso. Arch Argent Pediatr 2018;116(1):e82-e84. a. Turgut Özal Üniversitesi, Departamento de Pediatría, Angora, Turquía.

b. Turgut Özal Üniversitesi, Departamento de Pediatría, División de Neonatología, Angora, Turquía.

c. Turgut Özal Üniversitesi, Departamento de Radiología, Angora, Turquía.

d. Turgut Özal Üniversitesi, Departamento de Cirugía Pediátrica, Angora, Turquía.

Correspondencia:

Dra.Suzan Gunduz: akinsuzan@gmail.com

Financiamiento: Ninguno.

Conflicto de intereses: Ninguno que declarar.

Recibido: 7-1-2016

Aceptado: 12-7-2017

\section{INTRODUCCIÓN}

Los traumatismos obstétricos son una causa significativa de morbimortalidad neonatal. Entre los factores que incrementan el riesgo de traumatismos obstétricos se incluyen macrosomía (peso fetal mayor a 4000 g), obesidad materna, presentación podálica, parto vaginal instrumentado (es decir, con fórceps o ventosa), tamaño materno pequeño y presencia de anomalías pélvicas maternas. La incidencia general de traumatismos obstétricos es aproximadamente del $2 \%$ y del $1,1 \%$ en los partos vaginales únicos y por cesárea, respectivamente. El traumatismo obstétrico intrabdominal es poco frecuente y consiste básicamente de una ruptura o hemorragia subcapsular hacia el hígado, el bazo y las glándulas suprarrenales. ${ }^{1}$

El hematoma de la vaina de los rectos es la acumulación de sangre en la vaina del músculo recto mayor del abdomen y puede presentarse debido al daño directo al músculo propiamente dicho o al desgarro de uno de los vasos epigástricos superiores o inferiores que corren a lo largo de la cara posterior. Su etiología abarca traumatismo, cirugías, inyecciones de fármacos por vía subcutánea, tratamiento anticoagulante, enfermedades hemáticas, hipertensión, tos, ejercicio físico y embarazo; raramente, se produce de manera espontánea. El hematoma de la vaina de los rectos es una afección poco frecuente pero importante que causa dolor abdominal en los adultos. $^{2}$

En este artículo presentamos el caso de un recién nacido macrosómico con hematoma de la vaina de los rectos después de un parto distócico.

\section{A propósito de un caso}

Un bebé de sexo masculino de 4530 g nació por parto vaginal a las 40 semanas de gestación de una madre de 25 años (grávida: 2, para: 2); los puntajes de Apgar fueron 7 en el minuto $1 \mathrm{y}$ 9 en el minuto 5. Se trató de un parto distócico; se realizaron maniobras de extracción pero no se 
utilizaron fórceps ni ventosa. En los análisis de la madre no se observó coagulopatía y ella no estaba recibiendo anticoagulantes. Durante el examen físico inmediatamente después del nacimiento se observó un hematoma periumbilical de $1 \times 1 \mathrm{~cm}$ y un cefalohematoma de $1 \times 1 \mathrm{~cm}$ en el área parietoccipital. A las 3 horas de vida, la concentración de hemoglobina era de 13,6 g/ dl, el hematocrito era del $41 \%$, las plaquetas eran 191000 U/l, el tiempo de tromboplastina parcial activado (TTPa) era de 27,5 segundos, el tiempo de protrombina (TP) era de 15,7 segundos, la razón internacional normalizada (RIN) era de 1,23 y la bilirrubina total era de $8,9 \mathrm{mg} / \mathrm{dl}$. El resto de los análisis bioquímicos eran normales. En una ecografía abdominal se observó una masa tumoral de $66 \times 16 \mathrm{~mm}$ con líquido entre el ombligo y la porción inferior del esternón, que desplazaba los lóbulos derecho e izquierdo del hígado hacia la parte posterior (Figura 1). En una

FIgURA 1. En la ecografía en escala de grises se observa un hematoma hipoecoico en la parte delantera del lóbulo izquierdo del hígado (entre los cursores)

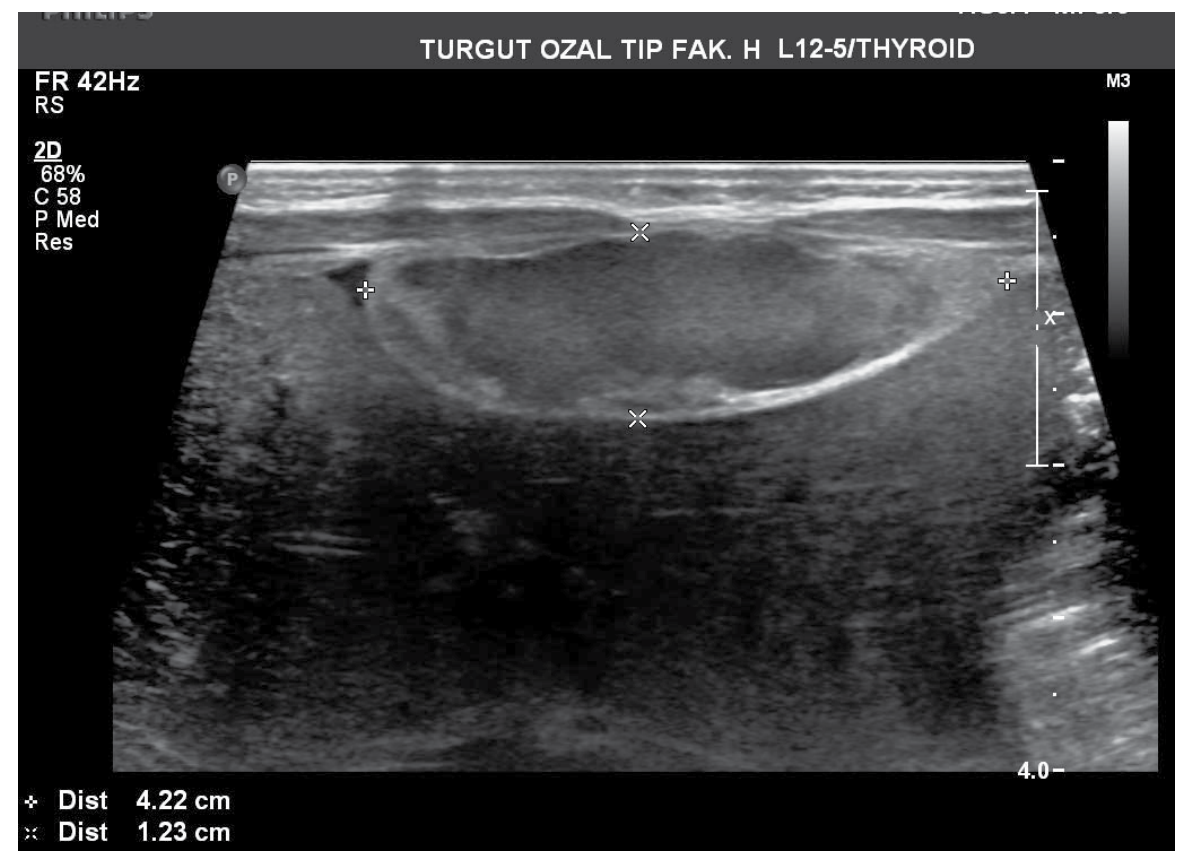

FIgURA 2. Plano transversal y plano frontal de tomografía computada del abdomen donde se observa hematoma de la vaina de los rectos

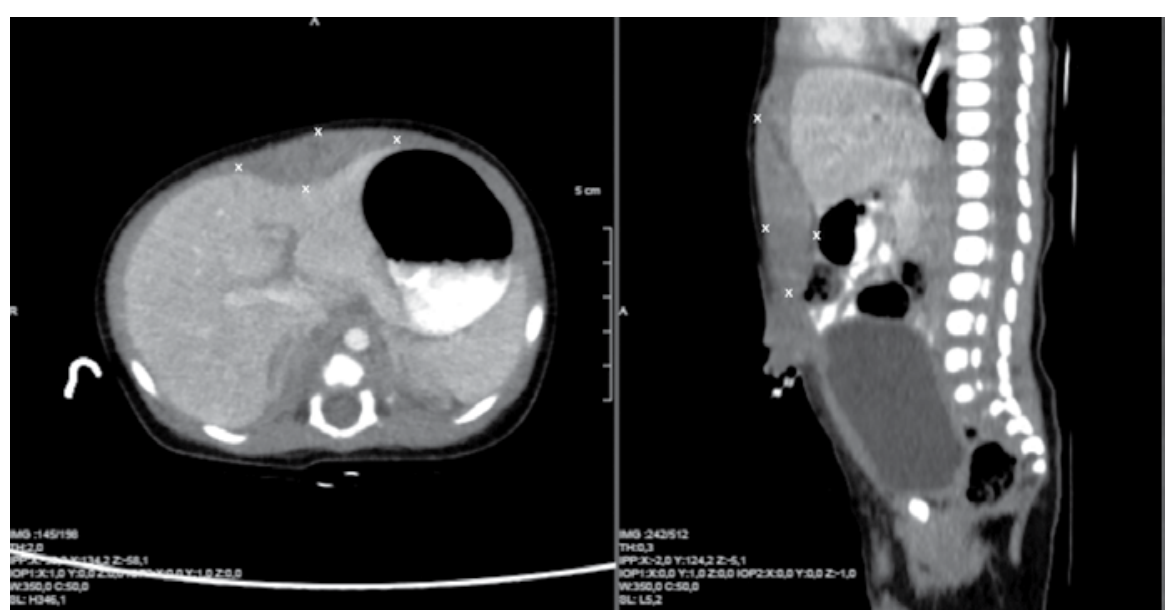


tomografía computada del abdomen se observó un hematoma de $7 \times 4 \times 2 \mathrm{~cm}$ en la vaina del recto derecho (Figura 2).

En la ecografía de los tejidos superficiales se encontró un cefalohematoma de $9 \times 9 \mathrm{~mm}$ en el área parietoccipital. En el hemograma completo a las 24 horas y a las 36 horas del ingreso, la hemoglobina era de $12,4 \mathrm{~g} / \mathrm{dl}$ y $14,3 \mathrm{~g} / \mathrm{dl}$, respectivamente, y la bilirrubina total era de $10,2 \mathrm{mg} / \mathrm{dl}$ y $10,8 \mathrm{mg} / \mathrm{dl}$, respectivamente. Se inició la lactancia después del nacimiento y no se requirió ningún otro tratamiento. Al paciente se le realizó un seguimiento frecuente mediante examen físico, hemograma completo y análisis de la concentración de bilirrubina, y no se observaron anomalías. Se le dio el alta a los 5 días de vida. Hoy tiene 5 meses de edad y no se observa ninguna patología en el examen físico ni en la ecografía abdominal.

\section{DISCUSIÓN}

El índice de traumatismos obstétricos es de alrededor del $2 \%$ en los partos vaginales normales con presentación cefálica y del $1,1 \%$ en las cesáreas. ${ }^{3}$ Los traumatismos obstétricos incluyen lesiones de los tejidos blandos (moretones, petequias, necrosis de grasa subcutánea, úlcera y perforación), cefalohematoma, tumor del parto, hemorragia intracraneal espontánea, lesión medular, lesión del plexo braquial (parálisis de Erb y parálisis de Klumpke), parálisis facial, lesión osteomuscular (fractura clavicular y tortícolis) y lesión isquémica hipóxica. ${ }^{4}$ En la mayoría de los estudios, el cefalohematoma y la fractura clavicular fueron los traumatismos obstétricos más frecuentes. ${ }^{5} \mathrm{La}$ incidencia de estos traumatismos ha disminuido gracias a los avances en la atención obstétrica y el diagnóstico prenatal. Sin embargo, la macrosomía es un factor de riesgo importante de traumatismo obstétrico; y el traumatismo obstétrico intrabdominal es poco frecuente. ${ }^{1}$ La ecografía es la mejor opción para diagnosticar los traumatismos obstétricos intrabdominales, y puede realizarse a pie de cama. La tomografía computada también sirve para obtener información diagnóstica útil.

Se debe observar al paciente para detectar posibles complicaciones, tales como ictericia, hipovolemia, choque hipovolémico, y establecer los tratamientos posibles para cada afección. El tratamiento del hematoma de la vaina de los rectos puede ser farmacológico o invasivo. La elección se determina según la condición del paciente. El manejo farmacológico es el tratamiento principal. Este consiste en reposo, analgesia, control de los factores predisponentes, compresión del hematoma, aplicación de hielo y, de ser necesario, neutralización de la anticoagulación, rehidratación o transfusión sanguínea y fototerapia para la ictericia. ${ }^{1}$ Debe considerarse el control de la hemorragia invasiva por el hematoma de la vaina de los rectos en los pacientes hemodinámicamente inestables que no responden a la rehidratación, cuyos hematomas se agrandan, o que muestran irritación peritoneal grave o signos de síndrome compartimental abdominal. ${ }^{6}$

Se realizó el seguimiento de este recién nacido; la lactancia se inició después del nacimiento y no se requirieron tratamientos médicos ni invasivos.

Si bien el hematoma de la vaina de los rectos se asocia con traumatismo, cirugías, inyecciones de fármacos por vía subcutánea y tratamiento anticoagulante en las personas de edad avanzada, en la bibliografía no se observaron casos en recién nacidos. ${ }^{7,8}$

Hasta donde sabemos, este es el primer caso de hematoma de la vaina de los rectos en un recién nacido macrosómico. El objetivo de la presentación de este caso era destacar el hematoma de la vaina de los rectos como traumatismo obstétrico en un recién nacido macrosómico. Los profesionales de la salud que se especializan en la atención neonatal deben considerar este traumatismo obstétrico poco frecuente en los recién nacidos macrosómicos.

\section{REFERENCIAS:}

1. Uhing MR Management of birth injuries. Pediatr Clin North Am 2004;51(4):1169-86.

2. Hatjipetrou A, Anyfantakis D, Kastanakis M. Rectus sheath hematoma: a review of the literature. Int J Surg 2015;13: 267-71.

3. Demissie K, Rhoads GG, Smulian JC, et al. Operative vaginal delivery and neonatal and infant adverse outcomes: population based retrospective analysis. BMJ 2004;329(7456):24-9.

4. Boulet SL, Alexander GR, Salihu HM, et al. Macrosomic births in the united states: determinants, outcomes, and proposed grades of risk. Am J Obstet Gynecol 2003;188(5):1372-8.

5. Warke C, Malik S, Chokhandre M, et al. Birth Injuries - A Review of Incidence, Perinatal Risk Factors and Outcome. Bombay Hosp J 2012;54(2):202-8.

6. Jafferbhoy SF, Rustum Q, Shiwani MH. Abdominal compartment syndrome: a fatal complication from a rectus sheath haematoma. BMJCase Rep 2012;2012:bcr 1220115332.

7. MaharajD, Ramdass M, TeelucksinghS, et al. Rectus sheath haematoma: a new set of diagnostic features. Postgrad Med J 2002;78(926):755-6.

8. Fitzgerald JE, Fitzgerald LA, Anderson FE, et al. The changing nature of rectus sheath haematoma: case series and literature review. Int J Surg 2009;7(2):150-4. 